\title{
Multiple Sclerosis Disease Modifying Therapy and Cancer risks
}

\author{
Schlindwein MAM, Chidambaram R, von Dokonal Ferreira AP and Gonçalves MVM \\ Department of Medicine, University of the Region of Joinville, Rua Ministro Calógeras, Bucarein, Joinville Santa \\ Catarina - 89202-207, Brazil
}

*Corresponding author: Gonçalves MVM, Department of Medicine, University of the Region of Joinville, Rua Ministro Calógeras, 439, Bucarein, Joinville, Santa Catarina - 89202-207, Brazil, Tel: +55 (47) 99666-3869, E-mail: mvmpesquisa@gmail.com

Citation: Schlindwein MAM, Chidambaram R, von Dokonal Ferreira AP, Gonçalves MVM (2020) Multiple Sclerosis Disease Modifying Therapy and Cancer risks. J Neurol Neurol Disord 6(1): 101. doi: 10.15744/24544981.6.101

Received Date: June 24, 2020 Accepted Date: December 28, 2020 Published Date: December 30, 2020

\begin{abstract}
Multiple sclerosis (MS) is a chronic inflammatory condition not commonly associated with cancer. Although since 1993 with the introduction of disease modifying therapies (DMTs), changing the natural history of the disease, cancer risk appears associated with some of these new therapies. We present the most common risks associated with each DMTs.
\end{abstract}

Keywords: Multiple Sclerosis, Disease Modifying Therapy; Cancer; Risk

\section{Introduction}

In multiple sclerosis (MS), a chronic inflammation of the central nervous system is the major component of the disease's pathology. If this is the major factor of the disease's pathogenesis, it's still a matter of debate nowadays [1,2]. The disease relapses were believed to be a $\mathrm{T}$ cell drive process, although recent evidence shows a growing importance of other cell types like B-cells and other peripheral myeloid cells [1,2], as well as CNS primary cells [2]. Once chronic inflammation is associated with cancer [3], a possible correlation between a chronic disease like multiple sclerosis and cancer have been studied throughout the time. There are studies showing decrease or no alteration in cancer risk of multiple sclerosis patients, even though some specific cancers show a different trend [4-8].

For example, a cohort study found a higher risk of malignant melanoma, although MS patients presented lower cancer risk and cancer mortality when compared to the general population [5]. An interesting fact found in another cohort was that even though MS patients had a lower incidence of cancer in comparison with the general population, they had an increased incidence of larger tumors. it points to the possibility of neglect diagnosis among MS patients [8].

In 1993, with the first disease modifying therapy (DMTs) for remittent relapsing MS, the natural history of the disease had been altered; but, with these, some new collateral effects and risk appeared. Among them, cancer risks [9,10]. As debated above, MS patients appear to have lower incidence of cancer, although a cohort study showed an increased risk only in patients exposed to immunosuppressant therapy [11]. MS patients that are not exposed to immunosuppressant therapy showed lower incidence of cancer, according with other studies [11]. This increased risk is particularly true for some drugs and some cancers that will be further discussed in separate sections $[9,10,12]$.

\section{Discussion}

\section{Fingolimod}

Fingolimod, a Sphingosine 1-P receptor modulator, complicates the egress of T and B cells from secondary lymphoid organs, causing peripheral lymphopenia. This S1P is involved in cell proliferation, migration, angiogenesis, inflammatory responses and lymphocyte trafficking, while sphingolipids such as ceramide and sphingosine are responsible for apoptosis. So, the dysregulation in sphingolipid metabolism allows cancer cells to escape cell death by increasing S1P signaling, altering expression of ceramide, degrading enzymes and increasing the sphingosine kinases, such as sphingosine kinase 1 (SK1) [9]. 
This drug has been associated in some clinical trials with skin cancer, mostly basocellular carcinoma [13], but also melanoma, squamous cell carcinoma and bowen's disease [13,14]. Those malignant neoplasms were reported in 4 patients receiving 0.5 mg of fingolimod, 4 receiving $1.25 \mathrm{mg}$ of fingolimod, and 10 receiving placebo. Besides that, the VIRGILE study, made in France, reported 8 cancers in 1023 patients treated with fingolimod [15]. Moreover, some evidence presented the risk of breast cancer, multiple myeloma, and, rarely, lymphoma [16-18]. So, it is necessary the screening of skin cancer once a year in patients that use this medication.

\section{Teriflunomide}

Teriflunomide is an imunossupressor that acts on the pyrimidine synthesis, reducing the lymphocytes count, and it has been used in MS treatment [10]. In two clinical trials, teriflunomide was associated with one case of cervical carcinoma 17 and one case of leiomyosarcoma [19], and they were reported in 4 different patients of the study. In a post marketing survey, it was found 308 cases of cancer, and breast and skin cancer were the most common ones [10]. In this case, it's important to keep screening MS patients in use of teriflunomide once a year, especially for skin cancer.

\section{Dimethyl Fumarate}

Dimethyl fumarate acts reducing the cytokines released during inflammation due to a lower immune cell activation, and it is also used in MS cases $[9,10]$. This drug increases the transcription of anti-inflammatory genes, and that's the expected effect in MS patients. It also provides an epithelial to mesenchymal transition, which leads to migration and metastasis. The dimethyl fumarate can also modify the metabolic environment in cancer cells, helping them to grow, survive and proliferate. In the clinical trials, Dimethyl fumarate was associated with low incidence of malignancies [9], even though a recent French post marketing survey from 2017 cumulated 32 reports of neoplasm. Those cases were breast (8) and skin (2 melanoma, 2 basocellular, 2 unspecified) [10].

\section{Cladribine}

Cladribine is a purinergic antimetabolite that acts on lymphocytes. It provokes lymphocyte apoptosis by inhibiting DNA synthesis and repair. First, cladribine causes sustained and fast reductions in numbers of CD4+ and CD20+ cells and temporary reduction in numbers of CD8+ and natural killer cells [9]. The use of this drug in patients with MS has shown 9 cases of uterine leiomyomas compared with 1 in the placebo group in a randomized control trial [19]. Despite that, a more recent study does not support the idea of cladribine increasing cancer risk even when compared to other treatments. This study called CLARITY showed a cancer rate of $0,34 \%$ and thus not different from all other groups of placebo-controlled trials, but further evidence is still needed [20].

\section{Natalizumab}

Natalizumab is an anti $\alpha 4$ integrin antagonist that blocks the attachment of cells in the brain endothelium, decreasing blood brain barrier inflammation [9]. Despite that, a recent study shows no increase in cancer risk in patients in use of natalizumab compared with the general population [21].

Melanoma risk increase is a fear surrounding the use of this drug. The Food and Drug Administration's (FDA) Adverse Event Reporting System (FAERS) reported 137 melanomas associated with natalizumab in MS patients [22], with one case of melanoma being reported in the clinical trial [9]. Besides that, a four-year observational study evaluating the risk of nevus transformation in melanoma in patients with MS in use of natalizumab showed no increased risk, and even proposed a protective effect [23]. Other cancers or precancerous lesions have been described in patients in use of natalizumab 9, like cervical dysplasia [24] and even a case of rapid progression of low-grade cervical dysplasia in an invasive cancer during natalizumab treatment [25].

\section{Alemtuzumab}

Alemtuzumab, a monoclonal antibody anti-CD52 that depletes B and T lymphocytes, has not been associated with an increase of cancer risk [10], although some cancer appears more frequently. In the clinical trials involving alemtuzumab, out of 1486 patients assigned, 29 (2\%) developed cancer. Thyroid papillary carcinoma (6) was the most frequent followed by basal cell carcinoma (6), breast cancer (5) and melanoma (4) [26]. Other malignancies had been described associated with alemtuzumab, including basal cell carcinoma, non-Epstein-Barr virus Burkitt's lymphoma and Castleman's disease [9].

\section{Rituximab and Ocrelizumab}

Rituximab is an anti-CD20 monoclonal antibody that is associated with cases of myelodysplastic syndrome, lymphoma and acute leukemia, but a relation between these cancers and multiple sclerosis has not been found [10]. In the Vygilize search, out of 3853 malignancies found in patients under Rituximab, 362 were myelodysplastic syndrome, 202 were acute leukemia and 197 were acute lymphoma [10]. Ocrelizumab, a humanized anti-CD20 antibody, has been linked to a variety of neoplasms. This drug was also the first effective medication for primary progressive MS. A study between 732 primary-progressive MS patients showed that, when in use of Ocrelizumab, the performance on the $25 \mathrm{ft}$ walk worsened in $38,9 \%$ of the patients, while the placebo rate was $51,1 \%$ [27]. The ORATORIO study showed that neoplasm occurred in $2.3 \%$ of the patients treated with ocrelizumab against $0.8 \%$ in the placebo group [27]. Patients using Ocrelizumab also have a higher risk of developing breast cancer [13], and once B cells have been linked with a better prognosis in breast and ovarian cancers B cells depletion therapy could have a worse outcome in these patients [9]. In the OPERA studies, 2 cases of breast cancer related to Ocrelizumab have been shown 8 and 4 in the ORATORIO trial [27]. 


\section{Interferon- $\beta$}

Interferon- $\beta$ was the first approved drug for MS treatment. Elevated blood levels of IFN- $\beta$ are associated with a reduced T-Cell activity, therefore increasing anti-inflammatory cytokines and reducing proinflammatory cytokines. Also, there is a reduced Th17 and neuroinflammatory actions [13]. This disease-modifying drug hasn't shown a significant increase of cancer risk, according to a french study using the European Database [10] and a in a British Columbia cohorts study [28]. However, in this cohort was found an increased risk in breast cancer among thus using interferon- $\beta$. Tumor size was similar between the treated and untreated group [28].

\section{Glatiramer Acetate}

Glatiramer Acetate action mechanisms are not well established. It seems to have an effect in shifting cytokines to anti-inflammatory responses. Also, there is APCs modulation and an increase in the neuroprotective factor 13. This drug is not very related to cancer either, and just one case of a primary lymphoma CD30+ was reported. Beyond that, these drugs can be used in patients with Multiple Sclerosis even if the patient has a cancer history. However, the follow-up with specific specialists is still important in cancer prevention [10].

\section{Authors Recommendations}

Once MS patients demand constant reevaluation by neurologists, it must be warned by this professional the risk involving MS treatment - especially cancer - to avoid negligence. Altering the patient prognosis, as it was discussed by Kingwell et al. [8].

Another important consideration when dealing with DMT in MS is the increase of cancer risk in patients that switched between more than one DMT [29]. This risk should be considerate when evaluating harm and benefits when a change in treatment is needed.

Our recommendations are: (1) All MS patients should receive multidisciplinary medical care; (2) Annual screening of skin cancers especially basocellular carcinoma in patients using fingolimod and teriflunomide [13-15,17]; (3) Annual screening of thyroid cancer in patients using alemtuzumab [10,26]; (4) Annual screening of breast cancer in patients using ocrelizumab [8,9,27]; (5) Annual screening melanoma and cervical cancer in patients using natalizumab [22,24,25]; (6) An special attention should be take in patients that had use more than one DMTs [29] (Table 1).

\begin{tabular}{|l|}
\hline \multicolumn{1}{|c|}{ Authors Recommendations } \\
\hline All MS patients should receive multidisciplinary medical care \\
\hline Annual screening of skin cancers especially basocellular carcinoma in patients using fingolimod and teriflunomide [13-15,17] \\
\hline Annual screening of thyroid cancer in patients using alemtuzumab [10,26] \\
\hline Annual screening of breast cancer in patients using ocrelizumab [8,9,27] \\
\hline Annual screening of melanoma and cervical cancer in patients using natalizumab [22,24,25] \\
\hline A special attention should be taken in patients that had use more than one DMTs [29] \\
\hline
\end{tabular}

Table 1: The Table presents the author's recommendations about the management of DMTs and cancer risk in MS patients

\section{Conclusion}

In conclusion, we advise neurologists to make sure their MS patients are constantly evaluated by dermatologists and gynecologists for active cancer surveys by most qualified specialists and analyze the risk-benefit balance of cancer risks when choosing or switching DMTs.

\section{Declarations}

In conclusion, we advise neurologists to make sure their MS patients are constantly evaluated by dermatologists and gynecologists for active cancer surveys by most qualified specialists and analyze the risk-benefit balance of cancer risks when choosing or switching DMTs.

\section{Author's Contributions}

Performed data acquisition and data analysis as well helping with the writing in the text: Marco Antônio Machado Schlindwein, Ramesh Chidambaram, Ana Paula von Dokonal Ferreira.

Made substantial contributions to conception and design of the study, as well as provided technical support: Marcus Vinicius Magno Gonçalves MD PhD.

\section{Copyright}

(C) Schlindwein MAM et al. 2020.

\section{References}

1. Filippi M, Bar-Or A, Piehl F, Preziosa P, Solari A, et al. Multiple sclerosis (2018) Nat Rev Dis Primers 4: 43.

2. Lassmann H (2019) Pathogenic Mechanisms Associated with Different Clinical Courses of Multiple Sclerosis. Front Immunol 9: 3116.

3. Murata M (2018) Inflammation and cancer. Environ Health Prev Med 23: 50. 
4. Moisset X, Perié M, Pereira B, Dumont E, Lebrun-Frenay C, et al. (2017) Decreased prevalence of cancer in patients with multiple sclerosis: A case-control study. PLoS One 12: e0188120.

5. Nørgaard M, Veres K, Didden EM, Wormser D, Magyari M (2019) Multiple sclerosis and cancer incidence: A Danish nationwide cohort study. Mult Scler Relat Disord 28: 81-5

6. Marrie RA, Reider N, Cohen J, Stuve O, Trojano M, et al. (2015) A systematic review of the incidence and prevalence of cancer in multiple sclerosis. Mult Scler 21: 294-304

7. Møller H, Kneller RW, Boice JD Jr, Olsen JH (1991) Cancer incidence following hospitalization for multiple sclerosis in Denmark. Acta Neurol Scand 84: 214-20.

8. Kingwell E, Bajdik C, Phillips N, Zhu F, Oger J, et al. (2012) Cancer risk in multiple sclerosis: findings from British Columbia, Canada. Brain 135: 2973-9.

9. Melamed E, Lee MW (2020) Multiple Sclerosis and Cancer: The Ying-Yang Effect of Disease Modifying Therapies. Front Immunol 10: 2954.

10. Lebrun C, Rocher F (2018) Cancer Risk in Patients with Multiple Sclerosis: Potential Impact of Disease-Modifying Drugs. CNS Drugs 32: 939-49.

11. Ragonese P, Aridon P, Vazzoler G, Mazzola MA, Re VL, et al. (2017) Association between multiple sclerosis, cancer risk, and immunosuppressant treatment: a cohort study. BMC Neurol 17: 155.

12. Kelm RC, Hagstrom EL, Mathieu RJ, Orrell KA, Serrano L, et al. (2019) Melanoma subsequent to natalizumab exposure: A report from the RADAR (Research on Adverse Drug events And Reports) program. J Am Acad Dermatol 80: 820-1.

13. Rommer PS, Zettl UK (2018) Managing the side effects of multiple sclerosis therapy: pharmacotherapy options for patients. Expert Opin Pharmacother 19: 483-98. 14. Guarnera C, Bramanti P, Mazzon E (2017) Comparison of efficacy and safety of oral agents for the treatment of relapsing-remitting multiple sclerosis. Drug Des Devel Ther 11: 2193-207.

15. Kappos L, Radue EW, O’Connor P, Polman C, Hohlfeld R, et al. (2010) A placebo-controlled trial of oral fingolimod in relapsing multiple sclerosis. N Engl J Med 362: 387-401

16. Schweitzer F, Laurent S, Fink GR, Barnett MH, Reddel S, et al. (2019) Age and the risks of high-efficacy disease modifying drugs in multiple sclerosis. Curr Opin Neurol 32: 305-12.

17. O'Connor P, Wolinsky JS, Confavreux C, Comi G, Kappos L, et al. (2011) Randomized trial of oral teriflunomide for relapsing multiple sclerosis. N Engl J Med 365: 1293-303.

18. Vermersch P, Czlonkowska A, Grimaldi LM, Confavreux C, Comi G, et al. (2014) Teriflunomide versus subcutaneous interferon beta-1a in patients with relapsing multiple sclerosis: a randomised, controlled phase 3 trial. Mult Scler 20: 705-16.

19. Cook S, Vermersch P, Comi G, Giovannoni G, Rammohan K, et al. (2011) Safety and tolerability of cladribine tablets in multiple sclerosis: the CLARITY (CLAdRIbine Tablets treating multiple sclerosis orallY) study. Mult Scler 17: 578-93.

20. Pakpoor J, Disanto G, Altmann DR, Pavitt S, Turner BP, et al. (2015) No evidence for higher risk of cancer in patients with multiple sclerosis taking cladribine. Neurol Neuroimmunol Neuroinflamm 2: e158.

21. Alping P, Askling J, Burman J, Fink K, Fogdell-Hahn A, et al. (2020) Cancer Risk for Fingolimod, Natalizumab, and Rituximab in Multiple Sclerosis Patients. Ann Neurol 87: 688-99.

22. Sabol RA, Noxon V, Sartor O, Berger JR, Qureshi Z, et al. (2017) Melanoma complicating treatment with natalizumab for multiple sclerosis: A report from the Southern Network on Adverse Reactions (SONAR). Cancer Med 6: 1541-51.

23. Pharaon M, Tichet M, Lebrun-Frénay C, Tartare-Deckert S, Passeron T (2014) Risk for nevus transformation and melanoma proliferation and invasion during natalizumab treatment: four years of dermoscopic follow-up with immunohistological studies and proliferation and invasion assays. JAMA Dermatol 150: 901-3.

24. Rolfes L, Lokhorst B, Samijn J, van Puijenbroek E (2013) Cervical dysplasia associated with the use of natalizumab. Neth J Med 71: 494-5.

25. Wan KM, Oehler MK (2019) Rapid Progression of Low-Grade Cervical Dysplasia into Invasive Cancer during Natalizumab Treatment for Relapsing Remitting Multiple Sclerosis. Case Rep Oncol 12: 59-62.

26. Havrdova E, Cohen JA, Horakova D, Kovarova I, Meluzinova E (2017) Understanding the positive benefit:risk profile of alemtuzumab in relapsing multiple sclerosis: perspectives from the Alemtuzumab Clinical Development Program. Ther Clin Risk Manag 13: 1423-37.

27. Montalban X, Hauser SL, Kappos L, Arnold DL, Bar-Or A, et al. (2017) Ocrelizumab versus Placebo in Primary Progressive Multiple Sclerosis. N Engl J Med 376: 209-20.

28. Kingwell E, Evans C, Zhu F, Oger J, Hashimoto S, et al. (2014) Assessment of cancer risk with $\beta$-interferon treatment for multiple sclerosis. J Neurol Neurosurg Psychiatry 85: 1096-02.

29. D’Amico E, Chisari CG, Arena S, Zanghì A, Toscano S, et al. (2019) Cancer Risk and Multiple Sclerosis: Evidence From a Large Italian Cohort. Front Neurol 10: 337.

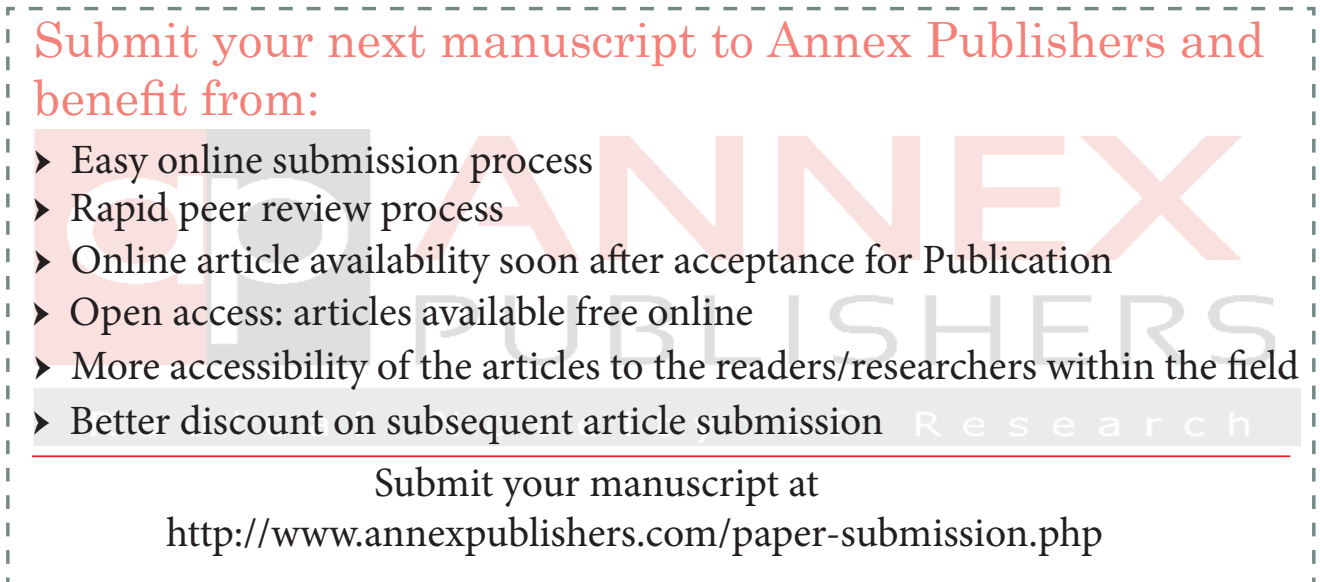

